Abstract: Experimental observation of the self-induced laser line sweeping (SLLS) in fiber ring lasers is presented. The SLLS with the same gain fiber is studied in Fabry-Perot cavity for comparison. The SLLS effect manifests itself as a laser wavelength drift with speed of the order of nanometer per second from shorter to longer wavelengths across several nanometers and fast backward jump. Recently, the dynamics of the SLLS in a Fabry-Perot cavity fiber laser was qualitatively described by a dynamic grating induced by spatial-hole-burning in the ytterbium doped fiber where the lifetime of the grating was related to the self-sustained relaxation oscillations. In this paper we address possible discrepancies between the published theoretical model and earlier observations of SLLS, particularly in fiber-ring lasers. We show that the qualitative theoretical model developed for explaining SLLS in the Fabry-Perot cavity can be used also to explain the SLLS effect we observed earlier in fiber-ring lasers.

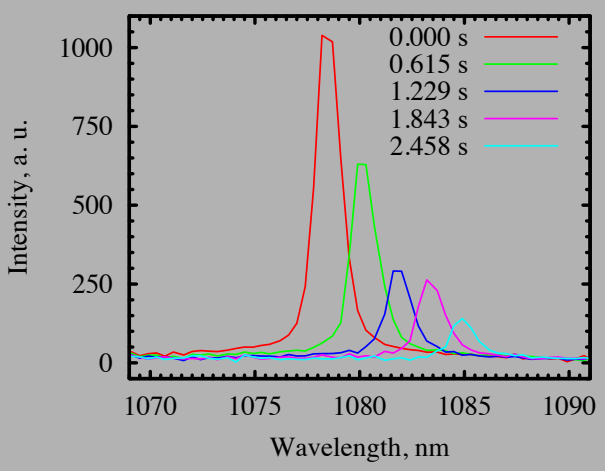

Video recording of the time drift of the laser wavelength of the Fabry-Perot fiber laser for pump power of $0.8 \mathrm{~W}$. The video was captured by the CCD-type spectrometer (video is available on-line).

\title{
Self-induced laser line sweeping in double-clad Yb-doped fiber-ring lasers
}

\author{
P. Peterka, ${ }^{1, *}$ P. Navrátil,,${ }^{1,2}$ J. Maria,${ }^{3}$ B. Dussardier, ${ }^{3}$ R. Slavík, ${ }^{1,4}$ P. Honzátko ${ }^{1}$ and V. Kubeček ${ }^{2}$ \\ ${ }^{1}$ Institute of Photonics and Electronics Academy of Sciences of the Czech Republic, Chaberská 57, 18251 Prague, Czech Republic \\ ${ }^{2}$ Czech Technical University, Faculty of Nuclear Sciences and Physical Engineering, Břehová 7, 11519 Prague, Czech Republic \\ ${ }^{3}$ Laboratoire de Physique de la Matière Condensée, CNRS - Université de Nice - Sophia Antipolis, 06108, Nice, France \\ ${ }^{4}$ Optoelectronic Research Centre, University of Southampton, Southampton, SO17 1BJ, United Kingdom
}

Received: January 2012, Revised: January 2012, Accepted: January 2012

Published online: 2012

Key words: fiber laser, ring cavity, long-period fiber grating, cladding pumping, ytterbium, tunable laser

PACS: 42.55 Wd, 42.60.Da, 42.60.Fc, 42.60.Mi, 42.60.Rn, 42.79.Dj, 42.81.-i

\section{Introduction}

In recent years we have been witnessing stunning achievements in rising power of cw fiber lasers and also in substantial increase of their share in global laser markets. On the other hand, rare-earth doped fiber lasers are subject to instabilities and various self-pulsation regimes which can lead to catastrophic damage of concerned components and relatively little work has been reported on these damage mechanisms. Particularly notorious in this regard are ytterbium-doped fiber lasers (YDFLs) [1]. The self- pulsation regimes have been attributed, e.g., to reabsorption in an unpumped part of the YDF [2], external perturbations like pump instabilities [3], scattering processes [4] and ion-pairs formation [5].

An interesting self-pulsing regime accompanied with laser line drift with time was observed in solid-state lasers in early days of lasers [6,7]. Despite several decades of intensive research of fiber lasers, the first detailed description of this so-called self-induced laser line sweeping (SLLS) in fiber lasers appeared only very recently [8]. The main characteristic of this effect is the self-scanning

*Corresponding author: e-mail: peterka@ufe.cz 
of laser line from shorter to longer wavelength, spanning over several nanometers, and its instantaneous bouncing backward. The time period of this sweeping is usually quite long, of the order of seconds. Kir'yanov and Il'ichev attributed this spectacular effect to spatial-hole burning (SHB) caused by standing-wave in the Fabry-Perot cavity. Kir'yanov and Il'ichev also noted that the effect was already mentioned in our previous work dealing with ringcavity YDFL [9]; with sweep achieved over a relatively broad range of $1076-1084 \mathrm{~nm}$.

Other important reports about the SLLS were published shortly after [8] by Lobach et al. [10,11]. They proposed a qualitative theoretical model of the SLLS and their theoretical findings correspond well to the experiments reported in the paper. However, there are two issues that need to be addressed. First, the fiber-laser in [8] is supposed to oscillate in continuous- or quasi-continuous-wave (cw) mode, while for the description in [10] the self-pulsing laser dynamics plays a fundamental role in the SLLS. The quasi-cw mode in [8] was described as "self-spiking with the characteristic times in a $1 \mu$ s range" and pulse energies were much weaker than those arising at the spectral bursts regime. This description can be in principle coherent with the self-sustained relaxation oscillations supposed in the model of Lobach et al. However it was not specified whether the SLLS was observed uniquely in quasi$\mathrm{cw}$ mode or also in truly cw mode. The other issue is that the SLLS effect reported in [9] deals with a unidirectional ring-cavity laser that generally does not involve any SHB. Therefore building of the dynamical grating in the cavity cannot be explained by the standing wave of the FabryPerot cavity and it is a question whether the recent explanations $[8,10]$ can describe the observed effect in fiberring lasers. In this paper we address the two above mentioned issues with particular emphasis on explaining the SLLS origin in fiber-ring laser cavities.

\section{Laser setups and the SLLS theory}

The effect of SLLS was observed up to now principally in two configurations of fiber lasers which are schematically shown in Fig. 2. The gain fiber was always an ytterbiumdoped double clad fiber. The Fabry-Perot setup in [8] differs from the one in Fig. 2a in that the GT-Wave ytterbium doped fiber bundle was used thus eliminating the need of the pump and signal combiner. In addition to the setup in Fig. 2a, Lobach et al. tested also a fiber-Bragg grating or fiber-loop-mirror as one of the Fabry-Perot resonator mirrors. The ring laser studied in [9] exploited one or more long-period fiber gratings (LPFGs) for selection of the laser wavelength or the ring laser was left lasing without any wavelength selective element.

In this paper we study both the Fabry-Perot and ring laser setups shown in Fig. 2. In both cases, the gain medium was a double-clad YDF (Liekki-nLight Yb12006/125DC) with nominal $2.6 \mathrm{~dB} / \mathrm{m}$ peak absorption in
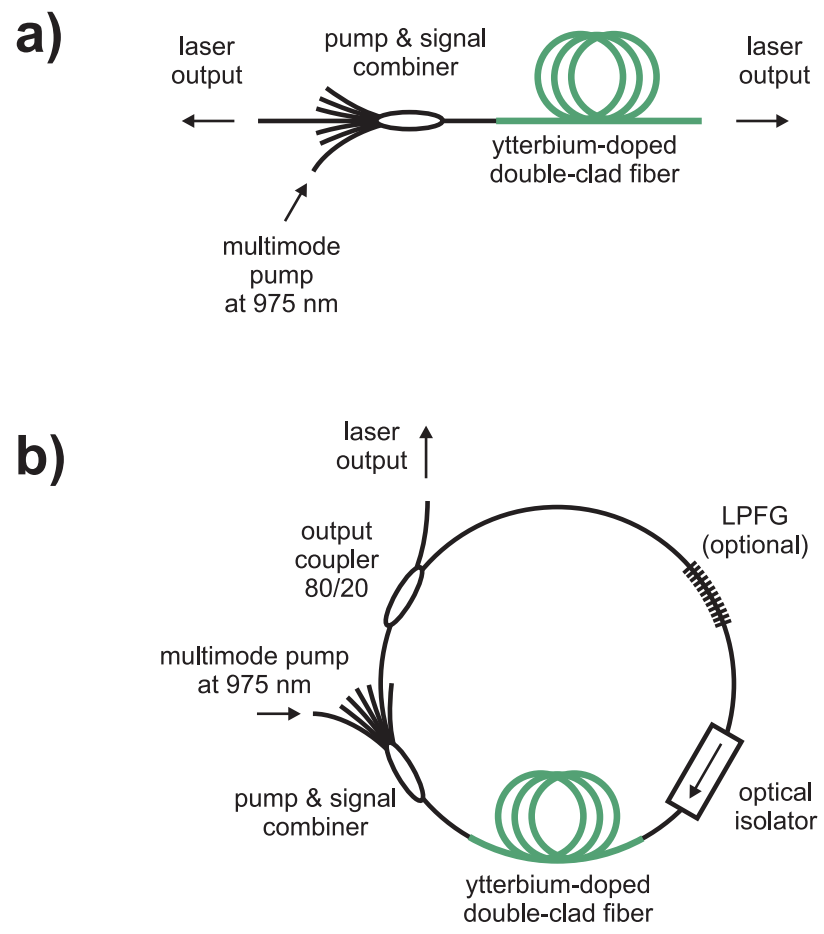

Figure 1 Setup of the fiber laser in Fabry-Perot (a) and ring configuration (b).

the inner cladding and $1200 \mathrm{~dB} / \mathrm{m}$ in the core. The inner cladding has an octagonal shape with $125 \mu \mathrm{m}$ average diameter and the core has the diameter of $6 \mu \mathrm{m}$ and numerical aperture of 0.13 . The outer cladding is made from low refractive index polymer. The laser output of the Fabry-Perot laser was obtained from perpendicularly cleaved fiber ends and in the case of ring YDFL the output was obtained from a fused optical fiber coupler. Usually, the $80 \%$ branch of the coupler was used for the output. A pigtailed optical fiber isolator was inserted into the ring cavity to ensure unidirectional propagation of the laser signal. The fiber isolator was the main lossy element in the cavity and as such it was placed behind the output coupler where the laser signal is minimal. Such a position also helped to prevent damage of the isolator by eventual selfQ-switched pulses. This configuration is commonly used for high-power fiber-ring lasers [3]. In the experiments, we used one multimode-pump laser diode pigtailed with multimode fiber of $105 \mu \mathrm{m}$ core diameter. Unless otherwise stated, the temperature of the pump laser diode was stabilized to $25^{\circ} \mathrm{C}$ and its central wavelength at maximum output power of $6 \mathrm{~W}$ is $975 \mathrm{~nm}$. The LPFG was inscribed onto the passive fiber (Fibercore PS1250/1500) by $\mathrm{CO}_{2}$ laser with the grating period of $177 \mu \mathrm{m}$ and length of $16.5 \mathrm{~mm}$ providing a band stop filter centered at $1070 \mathrm{~nm}$ with $3 \mathrm{~dB}$ and $10 \mathrm{~dB}$ suppression width of $32 \mathrm{~nm}$ and $18 \mathrm{~nm}$, respectively. Insertion of the LPFG into the ring resonator leads to modification of the net spectral gain of the laser. In such 
a way the operating wavelength of the ring laser can be selected in a wide range [9].

The SLLS effect was explained by Kir'yanov and Il'ichev by strong interference of narrow-linewidth, contra-propagating beams inside Fabry-Perot cavity. Superposition of the bouncing beams inside the Fabry-Perot cavity would create an interference pattern of nodes and antinodes in the YDF and consequently it would make a grating of higher signal gain (at nodes) and lower gain (at antinodes). This grating in turn causes movement of the longitudinal laser modes to the modes with better spatial overlap of higher gain areas in the YDF. From the spectral point of view the self-written grating exhibit itself as an inhomogeneous gain broadening. The gain/loss spectrum is then modulated by a function proportional to $\operatorname{sinc}\left(\mathrm{C}\left(\lambda-\lambda_{0}\right)\right)$ where the constant $\mathrm{C}$ depends primarily on the grating length and $\lambda_{0}$ is the wavelength of the narrow-bandwidth, high-power beam. The strength of the modulation depends on amplitudes of the interfering waves, rare-earth ions concentration, grating length and eventually on other factors. Such a spectral modulation is well known from applications in erbium doped fiber lasers, e.g., narrowing and stabilization of the linewidth of erbium doped fiber laser can be achieved by standing-wave saturation effect in an unpumped section of erbium doped fiber [12] or by SHB in twin-core fiber with cores doped with erbium [13].

Explanation of the sweeping dynamics was proposed by Lobach et al. [10]. Similarly to the early works on ruby lasers [7] they noticed that the SHB leads to self-sustained relaxation oscillations and therefore the laser generation occurs in the spiking mode. They observed square root dependence of both the sweep rate and frequency of selfpulsation on the laser output power. Based on this observation they linked the frequency of relaxation oscillations and the sweeping rate and they found that the sweeping rate is proportional to $\sqrt{P_{\text {out }} / L}$, where $P_{\text {out }}$ is the laser output power and $L$ is the cavity length.

\section{Experimental results}

For studying of the issue of the pulsed or cw operation of the SLLS, we have first built the Fabry-Perot YDFL according to Fig. 2a. The YDF length was $5 \mathrm{~m}$. For monitoring of the laser output spectra and temporal behavior we used one of the unused branches of the pump and signal combiner. About $0.5 \%$ of the laser signal is coupled into one pump branch of the combiner. The output spectrum was monitored by the CCD type spectrometer Ocean Optics HR2000CG-UV-NIR with $1 \mathrm{~nm}$ resolution. Under certain conditions, namely above laser threshold, we observed a drift of the laser wavelength with stable speed. The drift period is of the order of seconds; see the video sequence captured by the spectrometer in Fig. 2. The period of this particular SLLS is $3.24 \mathrm{~s}$. We detected the spectra also with a grating-monochromator-based optical spectrum analyzer (OSA) ANDO AQ-1425. The recorded

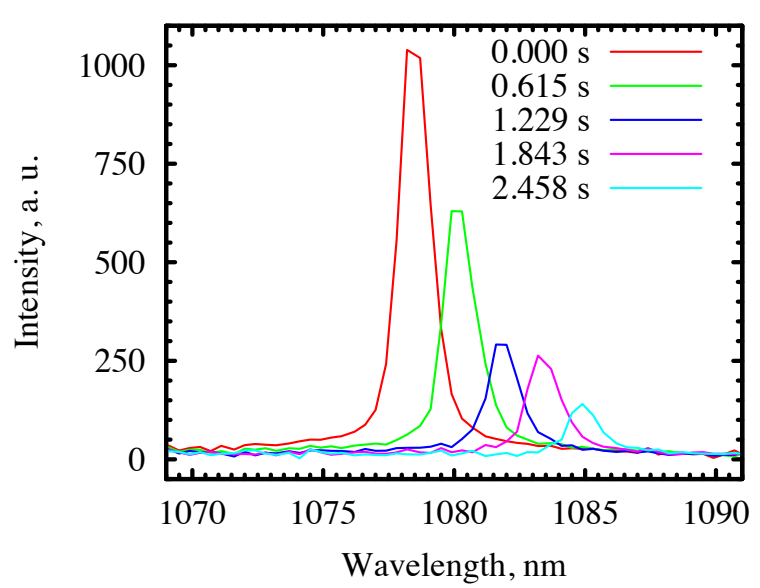

Figure 2 Video recording of the time drift of the laser wavelength of the Fabry-Perot fiber laser for pump power of $0.8 \mathrm{~W}$. The video was captured by the CCD-type spectrometer (video is available on-line).

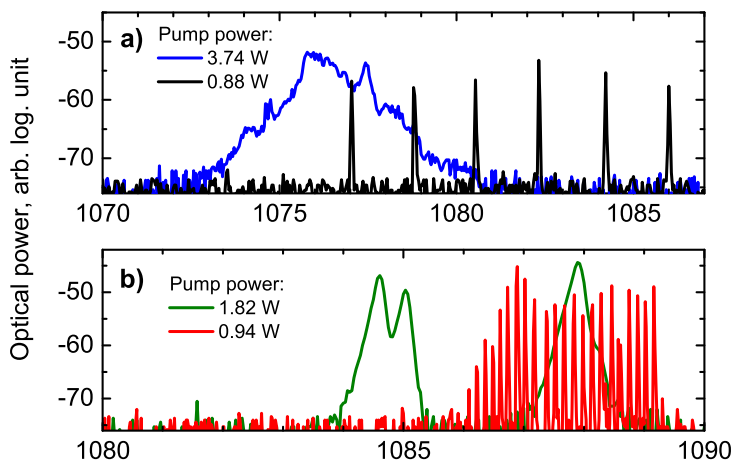

Figure 3 Fiber laser output spectra measured by the monochromator-based OSA: (a) Fabry-Perot and (b) ringlaser cavity.

spectra for several pump power levels are shown in Fig. 3a. The OSA resolution was set to $0.2 \mathrm{~nm}$. Measuring of one spectrogram in high-sensitivity mode of the OSA takes $44 \mathrm{~s}$. Therefore, the comb like structure of the spectra of $0.88 \mathrm{~W}$ of the pump power may indicate a periodic effect with period of the orders of seconds. The periodic effect is even more clearly manifested when the OSA is set to power meter mode, so that the output at a preset wavelength is recorded with time, see Fig. 4a. The resolution of the OSA was widened to $0.5 \mathrm{~nm}$ in order to avoid misdetection of the sweeping because of too narrow entrance slit of the OSA. The temporal behavior of the laser output was also detected by an InGaAs photodetector with 
1.2 GHz bandwidth and displayed by a digital oscilloscope (100 MHz bandwidth, $1 \mathrm{GSa} / \mathrm{s}$ ). Typical oscillogram of the SLLS regime is shown in Fig. 5a. Beating between neighboring longitudinal modes of the laser is apparent from the detail of one of the peaks in the inset of Fig. 5a. The separation of the longitudinal modes of $9.82 \mathrm{MHz}$ was detected. This value corresponds to a $10.5 \mathrm{~m}$-long cavity. The SLLS mode of operation was observed for pump power just above threshold to about $0.9 \mathrm{~W}$ as it is marked in the laser output characteristic vs. input pump power in Fig. 6. For higher pump powers, the SLLS regime disappeared and instead, a chaotic self-pulsation regime was established, with peak power higher than that of the peaks in Fig. 6 by several orders of magnitude. The characteristic pulse width was less than 10 ns.

For studying of the SLLS in fiber ring lasers, we added a fused fiber coupler and an isolator to the Fabry-Perot laser setup to form a ring cavity as shown in Fig. 2b. The laser outputs measured by the OSA are shown in Fig. 3b and Fig. 4b. No LPFG was included in the cavity. Similarly to the OSA measurements of the Fabry-Perot laser shown in Fig. 3a and Fig. 4a, the SLLS effect is clearly manifested even in the ring-laser setup. The SLLS effect disappeared for pump powers above $0.95 \mathrm{~W}$ and instead we observed competition of $\mathrm{cw}$ lasing at various wavelengths. It can be

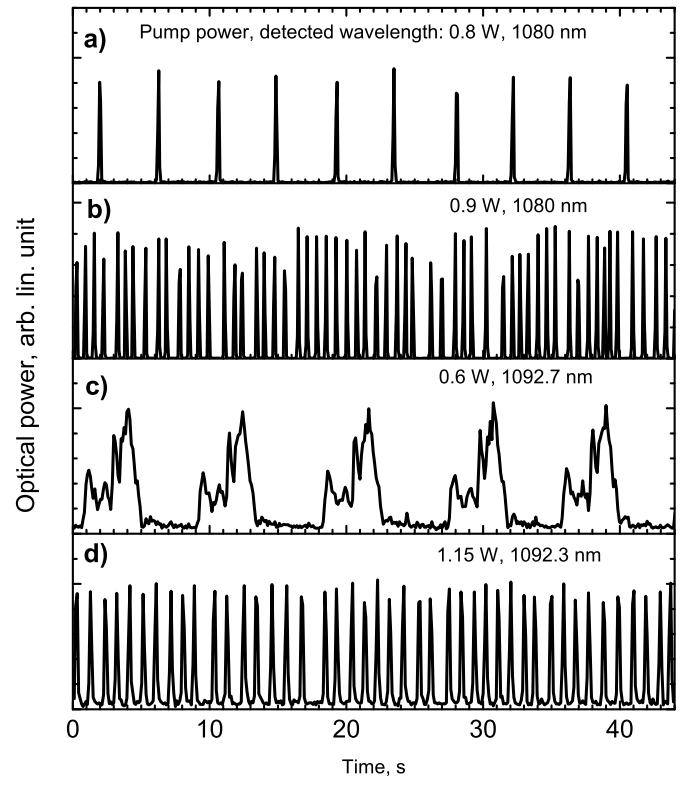

Figure 4 Fiber laser output measured by the monochromatorbased OSA in power-meter mode: (a) Fabry-Perot resonator, (b) ring-laser cavity, and (c, d) ring laser with LPFG. seen in the laser spectrum for the pump power of $1.82 \mathrm{~W}$ in Fig. 3b.
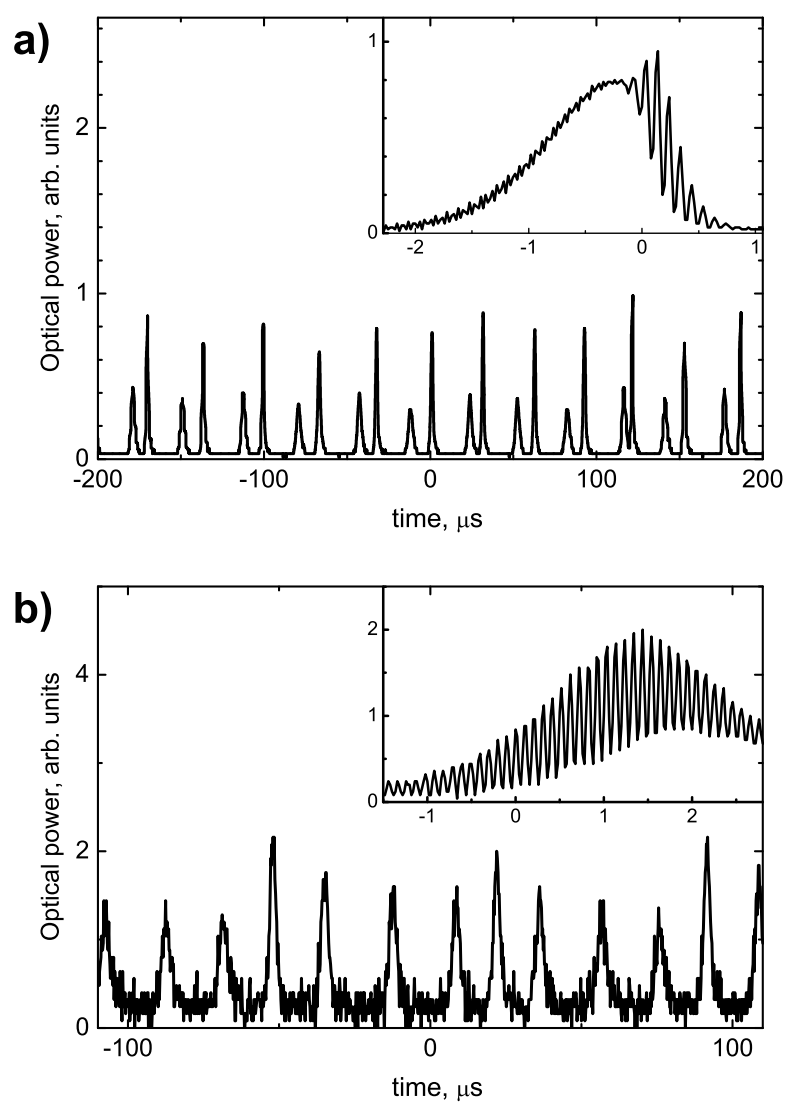

Figure 5 Oscillograms of the laser output: (a) Fabry-Perot resonator, pump power $0.6 \mathrm{~W}$, and (b) ring laser with LPFG, pump power $0.74 \mathrm{~W}$. The fine structure of pulses is shown in the insets.

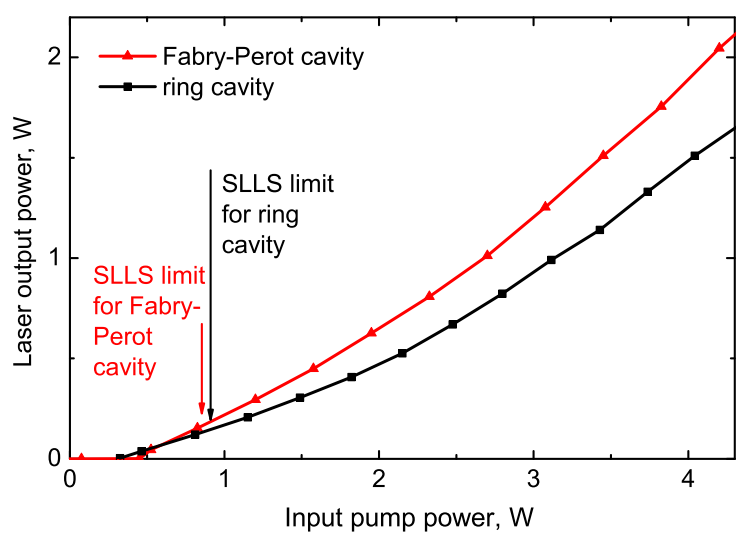

Figure 6 Output power characteristics versus pump power for various cavity configurations. The SLLS mode of operation exists up to pump power level depicted with an arrow. 


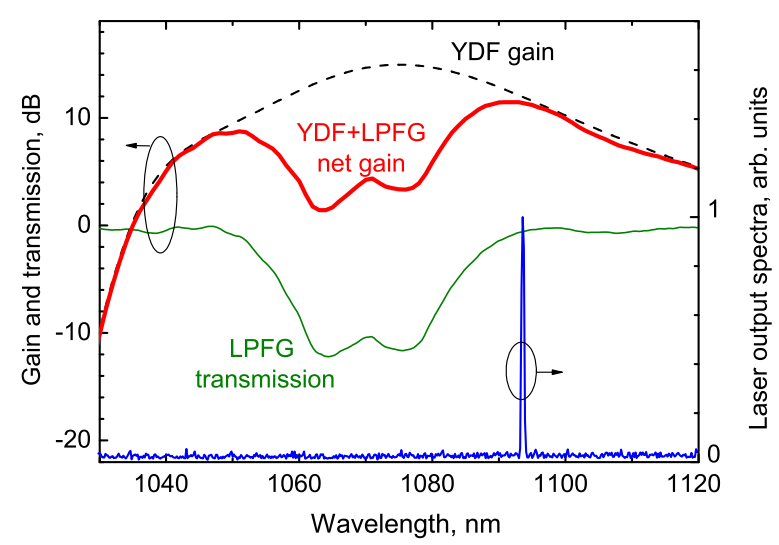

Figure 7 Transmission of the LPFG used in the ring laser and calculated gain of the YDF. The net gain bandwidth is limited by the LPFG. The position of the laser wavelength is on a side of attenuation band of the LPFG.

We also observed the effect of SLLS in the ring YDFL configuration with LPFG, though weaker than in the two preceding laser configurations. Incorporation of LPFGs into the fiber laser cavity can shift the laser wavelength, while preserving possibility of relatively wide laser-line sweeping range. This feature may be found useful in eventual application of SLLS where a specific wavelength range of sweeping would be needed. As an example we present evidence of SLLS in ring YDFL with one LPFG in the cavity. The laser setup is shown in Fig. 2b. The YDF length was $3 \mathrm{~m}$. The calculated gain profile of the combined YDF and LPFG is shown in Fig. 7 together with spectral characteristics of the LPFG sample and the calculated gain of the YDF itself. The laser output spectrum is also shown in the figure to pinpoint the position of the maximum cavity gain. The $3-\mathrm{dB}$ gain bandwidth of the combined YDF and LPFG is about $20 \mathrm{~nm}$, i.e., less than a half of the $3-\mathrm{dB}$ gain bandwidth of the YDF itself. The temperature of the pump laser diode was stabilized to $30^{\circ} \mathrm{C}$ in order to enhance the pump absorption in the shorter fiber at lower pump powers. The laser outputs measured by the OSA in power meter mode are shown in Fig. 4c for the pump power just above threshold and in Fig. 4d for the pump power close to the upper limit of SLLS regime. The observed wavelength range of SLLS was about $1 \mathrm{~nm}$. Typical oscillograms are shown in Fig. 5b. The temporal characteristics of the SLLS in Fig. $5 b$ are consistent with the two preceding laser configurations, i. e., the laser output is a train of $1-2 \mathrm{~s}$ long pulses envelopes with apparent beating of longitudinal modes of the ring laser cavity.

\section{Discussion}

The issue of the cw or pulsed operation of the SLLS regime of laser operation we studied in case of the FabryPerot and ring laser without LPFG. We have carefully scanned through the pump powers within the range of the SLLS and observed the time behavior of the laser output. We have never obtained cw mode of laser oscillation in this range. The laser was always in pulsed regime similar to that shown in Fig. 5a. This correspond well to the regime described as quasi-cw regime in [8] or as the selfsustained relaxation oscillations [10]. Our observations are consistent with the model proposed by Lobach et al.

The SLLS in a unidirectional fiber ring laser could have been attributed to various sustained self-pulsing instabilities or to temperature drift. For example, similar time period was observed in time fluctuations of interference fringes of the all-fiber Michelson interferometer used for measuring of the chromatic dispersion of optical fibers [14]. However, time period of these fluctuations changes in time or the fluctuations are not periodic at all, while the SLLS in ring lasers described here exhibits good long-term stability. It should be mentioned that the thermal effects as a cause of the SLLS in Fabry-Perot fiber lasers were excluded in discussion in [8].

Following the detailed reports and investigations for the origins of the SLLS in $[8,10]$, we have tried to find the parasitic Fabry-Perot cavity in our ring YDFL. Possible sources of reflections could be the non-ideal splices and perpendicularly cleaved ends of the output coupler and the pump and signal combiner. Although the main portion (about 89\%) of the laser signal is transmitted through the signal port of the combiner, about $0.5 \%$ of the laser signal arrives at each of the pump branches too and can be reflected. One of the main sources of reflection would be the perpendicularly cleaved end of the output coupler. Taking into account the transmission of the combiner and the coupler and neglecting the splice losses of the YDF and the combiner, about $1.5 \%$ of the laser output can be reflected back to the YDF. Indeed, after addition of a fiber terminated with an angle-polished connector to the $80 \%$ branch of the output we achieved $\mathrm{cw}$ regime of operation of the ring YDFL for the whole range of available pump power. In contrast to observation of SLLS in Fabry-Perot cavities, where the SHB is caused by standing wave between both cavity mirrors, in the case of the ring laser the standing wave responsible for SLLS was created by interference of the laser signal wave and its single weak reflection at the output coupler. It means that the interference between only two counter-propagating laser signal waves in the ring YDFL may lead to sufficient modulation of the ytterbium population inversion and create the dynamical grating responsible for the SLLS. 


\section{Conclusions}

We reported details about the observation of SLLS in three different fiber laser configurations: Fabry-Perot, ring without wavelength selective element and ring laser with LPFG. We have found that the SLLS effect in ring cavities can be explained also by creating a dynamical grating in longitudinal distribution of population inversion of ytterbium along the gain fiber as it was explained recently for the Fabry-Perot cavities. However, in contrast to the papers of Kir' yanov and Il'ichev and Lobach et al., the dynamical grating in the YDF was not created by the standing wave of the laser signal oscillating between the two mirrors of the Fabry-Perot cavity, but instead by relatively weak interference of the laser signal with reflection from the output coupler. We have observed that a sustained self-pulsing is a characteristic feature of the SLLS regime, which is consistent with the findings of Lobach et al. The turning-up of SLLS in fiber lasers may help laser designer to find source of possible parasitic reflections and sources of instabilities. Although the SLLS is an undesired effect in YDFL intended for $\mathrm{cw}$ mode of operation, this effect may find useful applications, e.g., in interrogation of optical fiber sensor systems. Indeed, the cavity and pump power regions of YDFL can be optimized to provide relatively wide and stable SLLS output. One particular advantage of such a swept source is its simplicity as it does not require any external driving electronics, apart from the pump laser diode driver.

Acknowledgments This work was supported by the French-Czech program Barrande (EGIDE-France, project No. 17360VA and Czech Ministry of Education, Youth and Sports, project No. ME10119); and by the European action COST TD1001.

\section{References}

[1] D. J. Richardson, J. Nilsson, and W. A. Clarkson, J. Opt. Soc. Am. B 27, B63 (2010).

[2] A. Hideur, T. Chartier, C. Özkul, and F. Sanchez, Opt. Comm. 186, 311 (2000).

[3] A. Hideur, T. Chartier, C. Özkul, and F. Sanchez, Opt. Lett. 26, 1054 (2001).

[4] B. N. Upadhyaya, A. Kuruvilla, U. Chakravarty, M. R. Shenoy, K. Thyagarajan, and S. M. Oak, Appl. Opt. 49, 2316 (2010).

[5] D. Marcuse, IEEE J. Quantum Electron. 29, 2390 (1993).

[6] T. P. Hughes and K. M. Young, Nature 196, 332 (1962).

[7] V. V. Antsiferov, V. S. Pivtsov, V. D. Ugozhaev, and K. G. Folin, Sov. J. Quantum Electron., 3(3), 211-215 (1973).

[8] A. Kir'yanov and N. Il'ichev, Laser Phys. Lett. 8, 305 (2011).

[9] P. Peterka, J. Maria, B. Dussardier, R. Slavik, P. Honzatko, and V. Kubecek, Laser Phys. Lett. 6, 732 (2009).

[10] I. A. Lobach, S. A. Babin, S. I. Kablukov, and E. V. Podivilov, In Proc. of 20th International Laser Physics Workshop (LPHYS'11), July 11-15 2011, Sarajevo, Bosnia and Herzegovina, p. 8.1.4.
[11] I. A. Lobach, S. I. Kablukov, E. V. Podivilov, and S. A. Babin, Opt. Express 19, 17632 (2011).

[12] Y. Cheng, J. T. Kringlebotn, W. H. Loh, R. I. Laming, and D. N. Payne, Opt. Lett. 20, 875 (1995).

[13] P. Peterka and J. Kanka, Opt. and Quantum Electron. 33, 571 (2001).

[14] P. Peterka, J. Kanka, P. Honzatko and D. Kacik, Opt. Appl. 38, 295 (2008). 